\title{
Les affordances culturelles : asymétries teintées de pouvoir
}

\author{
Mahault Albarracin, Mylène Legault et Jean-Nicolas Bourdon
}

\section{Résumé}

Les sciences cognitives et les sciences sociales n'ont pas atteint un niveau de connexion qui permet un échange paradigmatique suffisant pour développer adéquatement des modèles complexes. Grâce aux théories des affordances, les différentes disciplines peuvent trouver un point d'arrimage clément pour développer des modèles d'interactions sociales. Spécifiquement, il est possible de faire une analyse critique dans une optique féministe de la dynamique d'interaction entre des individus dans des groupes en opposition oppresseur/opprimé. Nous pouvons décliner ces interactions en termes d'asymétries d'affordances, déterminées par les niches culturelles (ou sociales), et en analyser les liens avec les injustices épistémiques.

Mots-clés : affordance, pouvoir, niche culturelle, injustices épistémiques

Les relations interpersonnelles sont empreintes de dynamiques de pouvoir. Les analyses de ces relations peuvent donc s'avérer complexes à réaliser et présentent le risque de considérer les personnes en situation de vulnérabilité comme des individus sans agentivité ${ }^{1}$ ou sans pouvoir. Elles peuvent aussi « sursimplifier » les dynamiques en jeu entre les individus, dans la vie de

\footnotetext{
${ }^{1}$ La notion d'agentivité est ici employée au sens proposé par le sociologue Anthony Giddens, qui la définit comme " everything in regard to which the subject has 'transformative power' (whether or not the subject ends up using it). Included in this definition was that somebody can have transformative power knowingly (the case of action) but also unwittingly (think, for example, of a small baby who starts crying in the middle of the night and thus instantly wakes up one or both parents and brings them to the cradle, or somebody who flies to conferences to promote social theory but ends up boosting climate change in the process). For Giddens, agency was thus a broader term than action and pointed to the need for analysing forms of power that are not well cognitively represented in the agent's (or anybody else's) mind. » (Heiskala, 2019, p. 53)
} 
tous les jours. En effet, les intersections entre différents rapports de domination ne sont pas nécessairement explicites. De même, les situations de marginalisation, de vulnérabilité ou d'oppression peuvent empêcher les personnes concernées de prendre conscience de leur situation, ce qui complexifie davantage l'analyse. Ces interactions sont pourtant observables et mesurables, notamment en tentant de comprendre la phénoménologie - c'est-à-dire l'expérience vécue - de ces interactions au niveau cognitif. Cela permet une meilleure compréhension des solutions à mettre en œuvre afin de modifier les dynamiques de pouvoir en place.

Dans cet article, nous tenterons de faire le pont entre les domaines des sciences sociales et des sciences cognitives. Ce faisant, nous prenons une approche féministe. Ceci nous permet d'avoir une approche critique sur des concepts généralement réservés à des domaines d'études plus positivistes, et associés à des pratiques d'oppression systémiques. Ainsi, nous élargissons les possibilités du féminisme en lui offrant les outils des sciences dures. Ces outils permettent de renforcer la crédibilité épistémique des études et des luttes féministes. Dans un premier temps, nous définirons les concepts liés aux sciences cognitives qui nous permettront de comprendre les éléments fondamentaux de notre objet d'étude, soit les affordances. Ensuite, nous détaillerons les différents niveaux d'affordance afin de jeter un éclairage sur la façon dont celles-ci s'inscrivent dans un contexte social. Nous expliquerons enfin en quoi ces systèmes d'affordances cristallisent des scripts ${ }^{2}$ sociaux asymétriques qui influencent à leur tour les affordances individuelles. En articulant les sciences cognitives et sociales, nous proposerons des pistes de réflexion pour de futures analyses et des pistes de solution pour pallier les asymétries de pouvoir mentionnées précédemment.

\section{Les affordances}

Les affordances sont des actions cognitives et comportementales à la jonction entre les habiletés développées par l'individu, ses capacités physiques et cognitives, et l'environnement

\footnotetext{
${ }^{2}$ Comme l'explique le sociologue Luc Van Campenhoudt, « [1] a théorie des scénarios sociaux (social scripts) montre que les comportements des individus en interaction obéissent à des scénarios préexistants où chaque partenaire a son rôle à jouer. Plus ces interactions sont délicates (comme une négociation difficile, une première relation sexuelle, un examen oral...) plus les partenaires se raccrochent à des scénarios précis et contraignants qui leur permettent de réduire leur incertitude. » (2014, p. 347)
} 
qu'il intègre (Almäng, 2008). Celles-ci nous donnent les outils pour comprendre la façon dont des situations se déclinent momentanément. Concrètement, une affordance est une possibilité qu'un individu perçoit, laquelle est médiée par les caractéristiques de l'individu et de l'environnement. Cette perception est donc relationnelle : par exemple, lorsqu'une personne voit un objet qu'elle sait utiliser, elle perçoit les possibilités liées à la façon dont elle sait qu'il fonctionne et dont elle est capable de l'utiliser. Ainsi, lorsqu'une personne donnée voit une tasse, elle voit l'affordance d'attraper la tasse par la hanse et d'y boire un liquide. Il en va de même pour des situations plus abstraites comme des interactions avec d'autres individus, qui sont encadrées par une variété de possibilités (Ramstead et al., 2016).

Conséquemment, les affordances peuvent se décliner à plusieurs niveaux. Elles sont déclinées aux niveaux temporels des script sociaux (par exemple, des séquences temporelles attendues) et des types d'interaction auxquels les affordances s'accrochent (par exemple, des types de situations en fonction des rôles que prennent les interlocuteurs) : il peut donc y avoir des affordances physiques, aussi appelées « schèmes sensorimoteurs » (Di Paolo et al., 2017) ; des affordances comportementales, lesquelles renvoient aux interactions avec des individus ; et des affordances culturelles, qui sont dictées par les normes des sous-groupes où se trouvent les individus (Ramstead et al., 2016). Les affordances peuvent s'agencer en scripts concrets de petite envergure (comme l'action de lever la main pour attraper des objets sur une étagère) ou, à l'inverse, de nature plus abstraite et complexe (comme une occasion de socialisation lors d'une soirée). Toutes les affordances peuvent être déclinées en séries d'affordances davantage restreintes dans le temps et dans l'espace, mais il est utile de considérer une affordance en relation à sa saillance (le fait que quelque chose retienne notre attention) reliée à un objectif. Par exemple, lors de la conduite automobile, il est plus utile de considérer l'affordance au niveau du passage de la voiture sur le feu rouge (niveau moins restreint dans le temps et l'espace) que du mouvement du pied vers la pédale (niveau plus restreint dans le temps et l'espace) si l'on reste au niveau spatio-temporel d'un voyage entre deux villes. 
La notion d'affordance permet de comprendre que les perceptions de la réalité sont reliées à notre position ancrée dans le monde (Almäng, 2008). Cela remet en question les compréhensions matérialistes de la réalité, selon lesquelles le monde extérieur est considéré comme prédéterminé et accessible objectivement sans relation à l'individu qui le perçoit (Barad, 2007). Le paradigme néo-matérialiste inscrit plutôt la construction de la réalité dans la phénoménologie, soit dans l'interaction entre la perception et le monde extérieur (Barad, 2007). Ce paradigme s'agence aussi particulièrement bien avec la notion d'intersectionnalité conceptualisée par la juriste Kimberlé Williams Crenshaw (1989/2005), laquelle «stipulates that the various systems of oppression - such as racism, patriarchy, capitalism, and heterosexuality - are interacting and co-constituting. ॥ (Cooper, 2016) Puisque les individus existent à l'intersection de différents axes d'oppression, leurs perceptions du monde témoignent de positions différentes les unes des autres (Samuels et Ross-Sheriff, 2008). Leurs interactions avec autrui peuvent aussi offrir des affordances conditionnées par leur position sur différents axes de pouvoir. L'adoption d'une perspective néo-matérialiste et intersectionnelle permet alors de développer une compréhension féministe des affordances.

\section{Les affordances conditionnées}

La culture et la position qu'y occupe l'individu constituent donc d'importants déterminants des différentes affordances perceptibles par celui-ci. Le sociologue Pierre Bourdieu propose la notion d'habitus pour expliquer comment les comportements sociaux ont un sens lisible par les personnes dans différents cercles communautaires. Cela encadre les vêtements, les pratiques, les

discours et tout autre marqueur socialement visible par des observateurs externes (Bourdieu, 1980/1990). Ainsi, différents éléments peuvent être observés par les individus afin de témoigner de leur statut social. Bourdieu (1980/1990) et Erving Goffman (1978) avant lui expliquent que l'environnement physique est utilisé pour «monter une scène » et offrir des référents sociaux qui permettent de comprendre les règles à suivre (et de percevoir les affordances correspondantes) afin de créer cette scène. Plus précisément, les objets mis en avant, les couleurs et la disposition de l'espace racontent une histoire sociale aux individus. Par exemple, dans un bureau de professionnel-les, les objets exposés peuvent témoigner de la compétence d'une 
personne dans son domaine. Dans le cas d'une maison, la plus grande chambre est souvent utilisée par les parents, reflétant ainsi leur rôle hiérarchique dans la famille. Dans le même ordre d'idées, dans un amphithéâtre, la scène se trouve à l'avant de la salle et les lumières y dirigent l'attention.

Si l'environnement proximal est ainsi une source importante de référents, les humains qui s'y trouvent jouent également un rôle important. Les individus dans des contextes sociaux participent aux mises en scène non seulement par leur façon de se présenter, mais aussi par leur façon d'interagir (Goffman, 1978). Par exemple, un sourire accueillant et un froncement de sourcils suggèrent habituellement des affordances différentes. De même, les bras croisés inspirent une attitude renfermée, là où les bras agités démontrent un état émotionnel actif. Les coupes de cheveux peuvent témoigner d'une appartenance à un groupe social, de même que le choix des vêtements portés. Pour les professionnel-les, les vêtements participent particulièrement à la compréhension du rôle d'une personne. Sans ces vêtements, le rôle ne serait pas aussi convaincant pour les collègues et son statut pourrait être remis en doute. Nous pouvons également prendre l'exemple des médecins qui, sans sarrau blanc dans les hôpitaux, n'inspireraient pas d'emblée la même autorité (Goffman, 1978). À partir de ces indices, les individus déterminent les comportements à adopter dans différents contextes.

Finalement, l'interaction entre les capacités physiques (et cognitives) et la structure de l'environnement joue un rôle crucial dans la détermination des affordances. Le modèle de corps considéré comme normatif est employé par plusieurs institutions comme état de base pour établir des normes de santé, de sécurité et de fonctionnement (Wehrle, 2016). Par exemple, la majorité des bâtiments sont construits en fonction des corps de personnes ayant la capacité de marcher ; la plupart des inscriptions sont conçues pour des gens qui peuvent lire avec leurs yeux ; ou encore, les sièges d'avion sont fabriqués pour des personnes d'une taille définie, pendant longtemps sans possibilité réelle de flexibilité. Toutes ces normes impliquent que les personnes dont les corps répondent aux critères d'inclusion normatifs ont accès à plus de ressources que les personnes dont les corps dévient de ces normes. Il en va de même pour les différents profils cognitifs qui 
sortent de la neuro-typicité. Dans la section qui suit, nous explorerons plus en détail le lien entre les affordances et le pouvoir.

\section{Le pouvoir en actions}

Nous avons jusqu'ici discuté d'affordances, mais qu'en est-il du pouvoir ? Le politologue Keith Dowding (2011) définit deux types de pouvoir qui nous seront utiles ici, soit le pouvoir sur les conséquences et le pouvoir social. Le premier renvoie à la capacité d'influencer le monde (environnement, société) afin d'obtenir le résultat que l'on désire, alors que le second représente la capacité d'influencer les agents dans le monde afin d'obtenir le résultat désiré. Ainsi, le pouvoir se décline sur un spectre spatiotemporellement varié d'une relation à l'autre, puisqu'il implique des ramifications différentes. Pour un agent, une des manifestations du pouvoir sur les conséquences est de chercher à atteindre un objectif par lui-même. L'agent est donc en contact direct avec l'affordance la possibilité perçue qui est liée à un objectif concret. Lier l'atteinte de ses objectifs à d'autres agents (soit le pouvoir social), c'est devoir modéliser les réactions de ces derniers et ajouter un certain degré d'incertitude à la réalisation de l'objectif. Cette stratégie n'est adaptative que dans le cas où les individus dont les réactions sont modélisées font partie d'une structure qui les rend prévisibles. Dans les cas contraires où les individus ne sont pas prévisibles, il est difficile de prévoir si l'on va atteindre son objectif, et donc de prendre les actions nécessaires. Cette distinction est pertinente puisqu'elle nous permet de souligner l'aspect parfois illusoirement désocialisé des relations de pouvoir. Nous reviendrons à cette distinction plus tard. Si l'on utilise la dyade du pouvoir social et sur les conséquences directes, le pouvoir sur les conséquences est en fait un ensemble d'affordances liées à des résultats dans le monde, tandis que le pouvoir social est un ensemble d'affordances associées à des interactions sociales qui sont à leur tour nouées à des affordances de l'ordre du pouvoir sur les conséquences.

Les individus manifestent leur pouvoir en enchaînant les affordances de façon à atteindre une série d'objectifs. Plus les objectifs d'une personne sont atteints, plus il est possible de penser qu'elle possède de l'agentivité et par conséquent du pouvoir, puisque l'agentivité relève précisément d'un « pouvoir transformateur » (voir la note 1). Cependant, avoir du pouvoir sur 
les autres n'implique pas nécessairement d'avoir des interactions sociales ou même que le pouvoir que l'on détient est directement social. Dans certaines situations, l'atteinte des objectifs équivaut plutôt à un jeu à somme nulle (zero-sum game) : plusieurs joueur-euses ne peuvent pas atteindre le même objectif, puisque l'un-e devra perdre pour que l'autre gagne (Andrejevic, 2010). Ainsi, même si l'obtention de certaines ressources au détriment d'autrui ne constitue pas nécessairement un acte social, cela entraîne un impact social.

Dans le cadre des affordances, cela implique que les individus doivent parfois se répartir sous la forme d'actions les affordances perceptibles; c'est ce qu'on appelle une " économie d'affordances ». La personne qui peut actualiser ces affordances réaliser le comportement - est celle qui atteindra ses objectifs. Un exemple plus concret serait la dernière part d'une pizza : si deux personnes veulent manger la dernière part de pizza et ne souhaitent pas partager, seulement l'une d'entre elles pourra en profiter et réaliser l'action souhaitée. La personne qui y aura accès sera donc celle qui aura atteint son objectif aux dépens de l'autre.

\section{L'asymétrie}

Les affordances reliées à des objectifs similaires chez deux ou plusieurs individus sont donc un jeu à somme nulle. Toutefois, les différents axes sociaux font en sorte que tous les individus ne sont pas sur un même pied d'égalité. En ce qui concerne le propos de cet article, le genre tel qu'envisagé par les normes sociales binaires dominantes définit les affordances des hommes différemment des affordances des femmes. Les normes, dans ce modèle, sont définies de façon à dessiner un ensemble supposément cohérent de caractéristiques complémentaires, spécifiques à chaque genre (Prudence, 2006).

Cette asymétrie d'affordances est intimement liée aux systèmes d'oppressions. Un groupe donné a accès à des affordances qui sont inatteignables pour d'autres groupes, ce qui teinte les réalités des individus qui en font partie. C'est ce refus d'affordances qui nous amène à parler d'asymétrie. En situant l'opprimé-e et l'oppresseur-e aux points opposés d'un ordre linéaire, la structure s'oppose à un ordre type impliquant la symétrie : il n'y a pas de ressemblance ni de similitude et surtout pas d'équivalence. L'un reste subordonné à l'autre. Ces systèmes sont 
insidieusement renforcés par eux-mêmes. En effet, si des affordances sont offertes à un groupe en priorité, celui-ci aura tendance à développer plus rapidement des habiletés qui leur sont reliées (Dohn, 2009), ce qui en retour justifiera que ces affordances soient accordées en priorité à ce groupe. Par exemple, dans les travaux manuels, plus une personne est considérée comme « naturellement » bonne (ce qui est souvent le cas des hommes), plus il lui sera proposé d'en effectuer. Ainsi, les habiletés manuelles de la personne pourront s'améliorer et on lui demandera d'en faire encore davantage. Dans le même ordre d'idées, plus on pousse les garçons à s'intéresser aux sciences « dures » à l'école et plus ceux-ci développent des habiletés dans ce domaine, renforçant la croyance qu'ils possèdent "naturellement " ces habiletés (Neu et Weinfeld, 2007). Au contraire, comme les filles y sont moins encouragées, elles auront moins la chance de développer ce type de compétences ni même la croyance qu'elles en sont capables (Archer et MacRae, 1991 ; Lane et al., 2012). Cela a pour effet de rendre l'économie d'affordances encore plus asymétrique. En outre, les emplois en STIM (sciences, technologies, ingénieries et mathématiques) sont beaucoup mieux payés que ceux du secteur des services et sont majoritairement occupés par des hommes cis et blancs, lesquels gagnent alors beaucoup plus d'argent que les femmes qui occupent la majorité des emplois de service (Beede et al., 2011 ; Hill, Corbett, et St Rose, 2010 ; Lahelma, 2000 ; Xu, 2015).

\section{La culture}

Ces distributions d'affordances peuvent sembler facilement réversibles, mais la cristallisation des croyances, soit le processus par lequel des observations anecdotiques deviennent des croyances qui façonnent notre perception de la réalité, fait en sorte que les normes et les pensées des individus sont complexes à modifier. De même que les garçons et les filles maintiennent certaines croyances envers leurs capacités intrinsèques dans différents domaines considérés comme genrés, la société fixe à son tour ces croyances et ces caractéristiques. Ce phénomène s'explique en psychologie par la menace du stéréotype (Fine, 2012 ; Lamont et al., 2015). Celle-ci renvoie au fait que les individus tendent à sous-performer une tâche lorsqu'ils sont exposés à certains stéréotypes avant son exécution. Le rappel du stéréotype suffit ainsi, par anxiété de performance, à réduire le taux de performance du groupe stéréotypé, auto-réalisant 
alors le stéréotype. Cette cristallisation de croyances par un groupe donné s'effectue, entre autres, au niveau linguistique.

Au niveau linguistique, le langage reflète des croyances culturelles profondes et entraîne l'intériorisation inconsciente des normes sociales reliées à des enjeux de genre, de race, de capacité et d'axes socio-économiques (Coles, 2015). Dans leur article "Implicit Biais : Scientific Foundations ", Anthony G. Greenwald et Linda H. Krieger (2006) indiquent la présence de biais inconscients reliés à des groupes sociaux, indépendamment de ce qu'une personne reconnaît. Ces biais sont entretenus par des schèmes métaphoriques ${ }^{3}$ intégrés dans la structure même de la cognition de l'individu et de ses conceptualisations, ce qui se reflète notamment dans le langage. Il est possible d'illustrer cela en analysant l'expression " lancer comme une fille », employée comme une insulte (voir "Throwing like a Girl: A Phenomenology of Feminine Body Comportment Motility and Spatiality »d'Iris Marion Young, 1980). Il est ici compris que les filles sont plus faibles que les garçons et qu'ainsi, la personne visée lance faiblement. En effet, les champs linguistiques autour de la féminité soulignent souvent des conceptions stéréotypées basées sur la maternité, la douceur ou la fragilité, de même que liées à la sphère du privé, à ce qu'il est nécessaire de cacher, à la possession et au corporel. Le féminin est donc relégué à des enjeux physiques et émotionnels, en opposition aux enjeux rationnels et intellectuels du masculin (Visser, 1996).

Dans un contexte néo-libéral patriarcal, ces caractéristiques ne permettent pas le même niveau d'affordances. Puisque les affordances constituent un jeu à somme nulle et qu'elles sont distribuées de façon à favoriser les caractéristiques " masculines », le fait de présenter des caractéristiques " féminines » peut être source de désavantage (Gerber, 1989). Par exemple, reprenant l'idée du care associée aux emplois de service, les femmes ont tendance à offrir un plus grand travail émotionnel, physique et cognitif dans toutes les sphères de leur vie que les hommes, tout en occupant aussi un travail rémunéré (England, 2005 ; Guy et Newman, 2004). Quant aux

\footnotetext{
${ }^{3}$ Il s'agit de métaphores qui nous permettent de faire des associations, et qui façonnent nos pensées.
} 
hommes, s'ils prennent cette charge de travail, ils seront loués pour leurs efforts - vus comme exceptionnels - et gagneront par le fait même du crédit social (Goodwin et Jasper, 2006).

Ces croyances se cristallisent en structures sociales genrées sont et renforcent certains modèles mentaux chez les individus. Cela agence une niche sociale (aussi appelée niche culturelle), soit « the set of social environments in which the focal individual has non-zero inclusive fitness » (Saltz et al., 2016, p. 351). Autrement dit, l'environnement renforce les croyances de l'individu et, en retour, celui-ci intègre ce que l'environnement conditionne (Constant et al., 2018). Cela a aussi pour effet de servir d'échafaudage pour les nouveaux individus, qui peuvent se référer à leur environnement pour développer des connaissances sociales. Cet échafaudage est en quelque sorte utile puisqu'il peut représenter certaines réalités. Par exemple, il est attendu des femmes qu'elles offrent un labeur émotionnel à leur entourage, ce qui, dans les faits, est accompli par une grande majorité d'entre elles (Guy et Newman, 2004). C'est de cette façon que les stéréotypes ont un aspect pernicieux, puisqu'ils peuvent correspondre à des phénomènes réels. Le problème survient lorsque le stéréotype structure à son tour la réalité en devenant une norme perçue comme un fait immuable et naturel, invisibilisant ainsi la nature socialement construite de ce qu'il représente.

\section{Les institutions en tant que niches culturelles}

Les niches sociales se déclinent, entre autres, en institutions régulatrices. Les gouvernements, les politiques adoptées, les écoles, les hôpitaux et les réglementations associées sont autant d'institutions qui cristallisent des notions de genre et qui accordent des affordances différentes en fonction de celui-ci (Ljungholm, 2017).

Prenons l'exemple d'un espace mixte institutionnel composé d'un nombre égal de femmes et d'hommes ${ }^{4}$, lesquel-les ont tous-tes le même objectif : prendre la parole. En considérant que nous ne pouvons écouter qu'une personne à la fois, la prise de parole est un jeu à somme nulle si deux personnes désirent parler simultanément. Prendre la parole, c'est se faire entendre, faire

\footnotetext{
${ }^{4}$ Comme nous parlons de stéréotypes imposés par la société, notre exemple conserve une logique de binarité.
} 
valoir sa perception de la réalité et, potentiellement, ses intérêts. Cela présuppose cependant la perception, puis l'actualisation, d'une affordance de prise de parole. Comme les normes renforcent cette affordance chez les hommes davantage que chez les femmes, celles-ci auront moins tendance à prendre la parole dans un contexte de mixité.

Il est possible que les hommes actualisent tout simplement l'affordance de la prise de parole parce qu'ils y sont plus habitués et qu'ils ont eu l'occasion de développer des habiletés en ce sens. Il est aussi possible que les hommes perçoivent plus souvent les affordances de prise de parole en considérant que certaines de leurs pensées valent d'être partagées, là où les femmes auront peut-être plus de doute sur ce qu'elles peuvent apporter. De la même façon, il est possible qu'une croyance sociale (même sous la forme d'un biais implicite) fasse en sorte que l'on accorde une plus grande valeur à une intervention masculine qu'à une intervention féminine, laissant ainsi sous-entendre qu'il est valable de couper la parole aux femmes. Dans le cas où un homme interrompt une femme, la possibilité et la capacité de prise de parole sont pourtant évidentes pour les deux personnes, puisque la femme était déjà en train de parler. Cela signifie que l'homme se sera saisi de l'affordance malgré le fait qu'elle était déjà occupée. Dans sa façon de gagner le jeu à somme nulle, l'homme perçoit l'économie d'affordance différemment de la femme. Selon lui, la prise d'affordance de la femme n'a pas le même poids que celle d'un autre homme. L'affordance lui reste donc partiellement ouverte. L'habileté à couper ou à prendre la parole peut ainsi être comprise comme étant plus développée chez les hommes, ce qui crée un taux inégalitaire d'intervention orale. Nous pouvons alors observer que la perception d'affordance est fortement modulée par la position sociale ; la position de privilège de l'homme façonne la façon dont il perçoit ses propres affordances et dont il les développe. Les interactions empreintes de pouvoir se déclinent en affordances partagées par un jeu à somme nulle asymétrique. L'exemple de la prise de parole est bien montré par Sue Montgomery, mairesse de l'arrondissement montréalais de Côte-des-Neiges-Notre-Dame-de-Grâce : pendant les assemblées du conseil municipal, l'élue tricotait avec des mailles rouges lorsque des hommes prenaient la parole et des mailles vertes lorsque des femmes faisaient de même. L'écharpe suivante, majoritairement rouge, en est ressortie : 


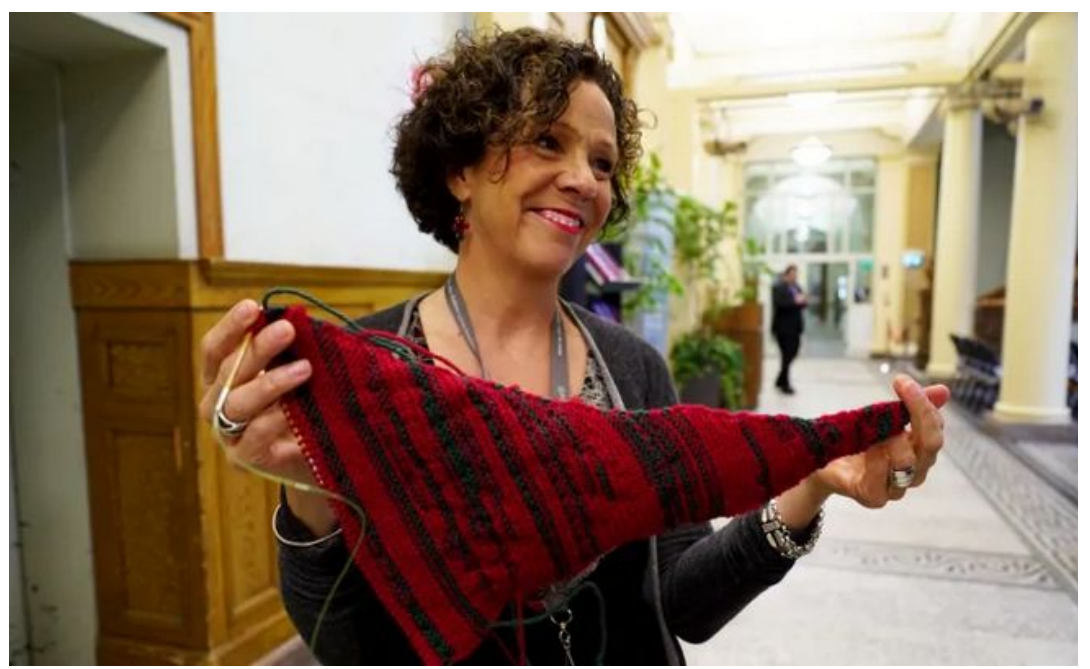

Figure 1. Sue Montgomery présente l'écharpe qu'elle a tricotée au fil des séances du conseil de la Ville de Montréal. Photographie de Charles Contant/CBC dans un article de Marilla Steuter-Martin (2019).

\section{Et l'agentivité épistémique ?}

Les affordances modulent l'accès épistémique de certains groupes d'individus, limitant ainsi leur capacité de changer les perceptions et croyances d'autres individus. Miranda Fricker (2007) est une figure clé de la littérature sur les injustices épistémiques, c'est-à-dire qui relèvent du domaine de la connaissance. L'auteure distingue les injustices testimoniales d'une part, puis les injustices herméneutiques d'autre part. Les injustices testimoniales renvoient à un déficit indu de crédibilité à la personne qui témoigne : la personne n'est pas crue, n'est pas prise au sérieux en raison de préjugés. Quant aux injustices herméneutiques, il s'agit plutôt d'une absence de concepts adéquats pour décrire et mettre en lumière certaines expériences. Par exemple, même si des personnes vivaient du harcèlement sexuel avant l'arrivée de ce terme, il leur était difficile de dénoncer leur expérience, car elles restaient limitées par le vocabulaire existant (Fricker, 2007). Les injustices épistémiques affectent la production, l'utilisation et la transmission de connaissances ; les groupes marginalisés ne participent pas aux ressources herméneutiques - ils ne définissent pas les normes et ne se retrouvent pas non plus dans la compréhension dominante de la société. 
Revenons à la question des affordances. On peut ainsi interpréter les injustices épistémiques comme des lacunes d'affordances épistémiques dues à des asymétries plus ou moins accentuées dans la structure des niches culturelles. Ainsi, les affordances perçues par les groupes sont façonnées par leurs positions de pouvoir, et par les métaphores qui entourent ces groupes. Un individu qui appartient au groupe genré masculin perçoit donc des affordances différemment d'un individu qui appartient au groupe genré féminin, et ces différences se propagent et se cristallisent dans des normes institutionnelles. Les individus appartenant à des groupes qui ont moins de pouvoir n'ont pas non plus le pouvoir de changer les connaissances à leur sujet. Les niches culturelles étant structurées autour des rôles sociaux, de l'expérience, des capacités, des traits, etc. d'un groupe au détriment d'un ou de plusieurs autres, cela restreint les affordances des groupes marginalisés et les rend moins en mesure d'influencer la structure de la niche culturelle dominante. Cela entraîne une boucle de rétroaction (comme celle mentionnée plus tôt avec l'exemple des compétences genrées liées au travail manuel ou aux STIM) qui cristallise encore davantage les injustices épistémiques et les différences d'affordances. 


\section{Conclusion}

Pour conclure, la théorisation des affordances se situe dans un virage paradigmatique (Engel et al., 2013) où la cognition et l'action sont devenues inséparables, s'articulant particulièrement bien avec un paradigme néo-matérialiste. Ce paradigme nous permet d'adopter un langage et une perspective féministes qui reconnaissent que la position sociale de l'individu a un impact sur sa perception, ouvrant la porte à l'intégration de modèles intersectionnels. Si l'agentivité épistémique se définit comme une production, une utilisation et une transmission de savoirs, alors la manifestation d'affordances liées à ses objectifs est contrainte par l'accès à la connaissance. Nous avons vu plus tôt que les affordances sont indissociables des dynamiques de pouvoir, ce qui signifie que les dynamiques de pouvoir affectent cette agentivité épistémique. Le point culminant est que les affordances concernent des possibilités d'action et non les actions en tant que telles. C'est ici qu'entrent en ligne de compte les oppressions systémiques (Anderson, 2012) : le simple fait de ne pas avoir accès à une possibilité d'action est une marginalisation. L'absence d'affordance est donc une injustice, indépendamment de l'exécution de l'action.

Les injustices épistémiques, quant à elles, interfèrent dans les possibilités d'actions offertes par l'autre, puisqu'une personne d'un groupe opprimé ne se verra pas présenter les mêmes possibilités par des membres de groupes qui ont plus de pouvoir. Inclure la question des affordances au sein des injustices épistémiques vient renforcer une conception à la fois agentielle et systémique des injustices, tout en permettant de penser les affordances dans une dynamique relationnelle. Il est donc possible de penser plus profondément aux rôles des individus dans leur réitération du pouvoir et dans leur responsabilité face au changement.

Bien que cet article souligne différents niveaux auxquels se jouent les sources d'affordance, celles-ci sont principalement des processus d'ordre cognitif. Lorsque l'on prend conscience de ces processus, il est possible de les influencer. La conscientisation des différents groupes aux affordances disponibles, ainsi qu'aux relations de pouvoir qu'elles impliquent, permet de rendre la distribution des affordances plus équitable. Sensibiliser les personnes en position de pouvoir au fait que prendre la parole, par exemple, est une question d'affordance qu'elles perçoivent peut 
remettre en perspective leur position. Cela permet de souligner que l'affordance en question n'est pas fondamentalement la leur, mais qu'elle est prise au détriment d'autres individus. De même, faire remarquer aux personnes en situation d'oppression que des affordances leur sont disponibles peut leur offrir des possibilités d'actions jusqu'ici non envisagées. Nous croyons que la prise en compte des asymétries d'affordances permet de mieux penser des changements concrets pour le futur dans les ressources socioculturelles (comme l'accès au changement épistémique) afin d'assurer que la saillance des affordances soit égalitaire pour tous les groupes (dans le cas de notre sujet, elle devrait être accrue pour les femmes). Des études futures devraient se pencher sur l'efficacité de telles solutions dans les perceptions d'affordances par les différents groupes. 


\section{BIBLIOGRAPHIE}

Almäng, Jan. 2008. « Affordances and the Nature of Perceptual Content ». International Journal of Philosophical Studies, vol. 16, $\mathrm{n}^{\circ}$ 2, p. 161-177.

Anderson, Elizabeth S. 2012. « Epistemic Justice as a Virtue of Social Institutions ». Social Epistemology, vol. 26, nº 2, p. 163-173.

Andrejevic, Mark. 2010. «Social Network Exploitation». Dans Zizi Papacharissi (dir.), $A$ Networked Self: Identity, Community, and Culture on Social Network Sites. Londres : Routledge, p. 90-110.

Archer, John et Margaret MacRae. 1991. «Gender-Perceptions of School Subjects Among 10 -11 Year-Olds ». British Journal of Educational Psychology, vol. 61, nº 1, p. 99-103.

Barad, Karen. 2007. Meeting the Universe Halfway: Quantum Physics and the Entanglement of Matter and Meaning. Durham : Duke University Press, 544 p.

Baxter, Judith. 2002. « Jokers in the Pack: Why Boys Are More Adept Than Girls at Speaking in Public Settings ». Language and Education, vol. 16, nº 2, p. 81-96.

Beede, David, Tiffany Julian, David Langdon, George McKittrick, Beethika Khan et Mark Doms. 2011 (août). « Women in STEM: A Gender Gap to Innovation ». Economics and Statistics Administration Issue Brief, $\mathrm{n}^{\circ}$ 04-11, 11 p.

Bourdieu, Pierre. (1990). « Structures, Habitus, Practices ». Dans The Logic of Practice. (Richard Nice, trad.). Stanford : Stanford University Press, p. 52-65. 1980.

Coles, Alf. 2015 (avril). « On Enactivism and Language: Towards a Methodology for studying Talk in Mathematics Classrooms ». ZDM, vol. 47, $\mathrm{n}^{\circ}$ 2, p. 235-246.

Constant, Axel, Maxwell J. D. Ramstead, Samuel P. L. Veissière, John O. Campbell, et Karl J. Friston. 2018 (avril). « A Variational Approach to Niche Construction ». Journal of The Royal Society Interface, vol. 15, n 141, 14 p.

Cooper, Brittney. 2016. «Intersectionality ». Dans Lisa Disch et Mary Hawkesworth (dir.), The Oxford Handbook of Feminist Theory. New York : Oxford University Press, p. 385-406. 
Crenshaw, Kimberlé Williams. (2005). « Cartographies des marges : intersectionnalité, politique de l'identité et violences contre les femmes de couleur ». (Oristele Bonis, trad.). Cahiers du genre, vol. 2, nº 39, p. 51-82. 1989.

Di Paolo, Ezequiel, Thomas Buhrmann et Xabier Barandiaran. 2017. Sensorimotor Life: An Enactive Proposal. New York : Oxford University Press, 304 p.

Dohn, Nina Bonderup. 2009. " Affordances revisited: articulating a Merleau-Pontian view ». International Journal of Computer-Supported Collaborative Learning, vol. 4, n², p. 151-170.

Dowding, K. (dir.). 2011. Encyclopedia of power. Sage Publications.

Engel, Andreas K., Alexander Maye, Martin Kurthen, et Peter König. 2013. "Where's the action? The pragmatic turn in cognitive science ». Trends in Cognitive Sciences, vol. 17, n 5 , p. 202-209.

England, Paula. 2005. « Emerging Theories of Care Work». Annual Review of Sociology, vol. 31, p. 381-399.Récupéré de January 31, 2020, from www.jstor.org/stable/29737725

Fricker, Miranda. 2007. « Epistemic Injustice: Power and the Ethics of Knowing ». Oxford University Press.

Fine, Cordelia. 2012. « Explaining, or Sustaining, the Status Quo? The Potentially Self-Fulfilling Effects of 'Hardwired' Accounts of Sex Differences ». Neuroethics, vol. 5, p. 285-294.

Gerber, Gwendolyn. 1989. "The more positive evaluation of men than women on the gender-stereotyped traits». Psychological Reports, vol. 65, nº 1, p. 275-286.

Goffman, Erving. 1978. The presentation of self in everyday life (p. 56). London: Harmondsworth.

Greenwald, Anthony. G. et Linda H. Krieger. 2006. "Implicit bias: Scientific foundations ». California Law Review, vol. 94, no 4, p. 945-967.

Guy, Mary Ellen, \& Newman, Meredith. A. 2004. « Women's jobs, men's jobs: Sex segregation and emotional labor ». Public administration review, vol. 64, n³, p. 289-298.

Heiskala, Risto. 2019. « On agency/power, meanings/materialities, and political conjuncture in the US and Europe: Comments on Reed and Weinman ». European Journal of Cultural and Political Sociology, vol. 6, $\mathrm{n}^{\circ} 1$, p. 51-67. 
Hill, Catherine., Corbett, Christianne, et St Rose, Andresse. 2010. « Why so few? Women in science, technology, engineering, and mathematics ». American Association of University Women. 1111 Sixteenth Street NW, Washington, DC 20036.

Lahelma, Elina. 2000. « Lack of male teachers: A problem for students or teachers? ». Pedagogy, Culture and Society, vol.8, n 2 , p. 173-186.

Lamont, Ruth. A., Swift, Hannah. J., \& Abrams, Dominic. 2015. « A review and meta-analysis of age-based stereotype threat: Negative stereotypes, not facts, do the damage ». Psychology and Aging, vol. 30, nº 1, p. 180-193. http://dx.doi.org/10.1037/a0038586

Lane, Kristin. A., Goh, Jin. X., \& Driver-Linn, Erin. 2012. « Implicit science stereotypes mediate the relationship between gender and academic participation ». Sex Roles, vol. 66, $\mathrm{n}^{\circ} 3-4$, p. $220-234$.

Ljungholm, Doina Popescu. 2017. «Feminist institutionalism revisited: The gendered features of the norms, rules, and routines operating within institutions ». Journal of Research in Gender Studies, vol.7, nº1, p. 248-254.

Neu, Terry. W., \& Weinfeld, Rich. 2007. «Helping boys succeed in school: A practical guide for parents and teachers ».

Owens, Lynn et Jasper, James. 2006. « Emotions and social movements ». Dans Handbook of the Sociology of Emotions (p. 611-635). Springer, Boston, MA.

Prudence, Allen. 2006. « Man-woman complementarity: The Catholic inspiration ». Logos : A Journal of Catholic Thought and Culture, vol. 9, n³, p. 87-108.

Ramstead, Maxwell. J., Veissière, Samuel. P., \& Kirmayer, Laurence. J. 2016. « Cultural affordances: scaffolding local worlds through shared intentionality and regimes of attention ». Frontiers in Psychology, vol. 7, p. 1090.

Saltz, Julia.B., Geiger, Adam. P., Anderson, Raleigh., Johnson, Benjamin., \& Marren, Rachel. 2016. "What, if anything, is a social niche? ». Evol Ecol vol.30, p. 349-364. https://doi.org/10.1007/s10682-015-9792-5

Samuels, Gina. Miranda., \& Ross-Sheriff, Fariyal. 2008. « Identity, oppression, and power: Feminisms and intersectionality theory ». Journal of Women and Social Work, Vol. 23, $\mathrm{n}^{\circ} 1, \mathrm{p} .5-9$ 
Steuter-Martin, Marilla. Mai 2019. Sue Montgomery calls out gender disparity at city council, one stitch at a time. Récupéré de https://www.cbc.ca/news/canada/montreal/montreal-city-council-gender-sue-montgomery $\underline{-1.5135001}$

Van Campenhoudt, Luc. 2014. « L’instant fugitif où la sociologie prend. Pour une sociologie du malentendu ». Dans Jean-Pierre Delchambre (dir.), Le sociologue comme médiateur ? Accords, désaccords et malentendus : Hommage à Luc Van Campenhoudt. Bruxelles : Presses de l’Université Saint-Louis, p. 475-506. Récupéré de http://books.openedition.org/pus1/5019

Visser, Irene. 1996. « The prototypicality of gender. » Women's Studies International Forum, vol. 19 , nº , p. 589-600. doi :10.1016/s0277-5395(96)00064-7

Wehrle, Maren. 2016. « Normative Embodiment. The Role of the Body in Foucault's Genealogy. A Phenomenological Re-Reading ». Journal of the British Society for Phenomenology, vol. $47, \mathrm{n}^{\circ} 1$, p. $56-71$.

$\mathrm{Xu}$, Yonghong. 2015. " Focusing on women in STEM: A longitudinal examination of gender-based earning gap of college graduates ». The Journal of Higher Education, vol. $86, n^{\circ} 4$, p. 489-523.

Young, Iris. Marion. 1980. « Throwing like a girl: A phenomenology of feminine body comportment motility and spatiality ». Human Studies, vol. 3, n 1, p. 13-156. 